STREET OCCUPATIONS 
THIS PAGE INTENTIONALLY LEFT BLANK 


\section{STREET OCCUPATIONS}

Urban Vending in Rio de Janeiro, 1850-1925

PATRICIA ACERBI 
Copyright (C) 2017 by the University of Texas Press

All rights reserved

Printed in the United States of America

First edition, 2017

Requests for permission to reproduce material from this work should be sent to:

Permissions

University of Texas Press

P.O. Box 7819

Austin, TX 78713-78I9

http://utpress.utexas.edu/index.php/rp-form

(a) The paper used in this book meets the minimum requirements of ANSI/NISO Z39.48-I992 (RI997) (Permanence of Paper).

LIBRARY OF CONGRESS CATALOGING-IN-PUBLICATION DATA

Names: Acerbi, Patricia, author.

Title: Street occupations : urban vending in Rio de Janeiro, I850/1925 /

Patricia Acerbi.

Description: First edition. I Austin : University of Texas Press, 20I7. I

Includes bibliographical references and index.

Identifiers: LCCN 2016052407

ISBN 978-I-4773-I355-8 (cloth : alk. paper)

ISBN 978-I-4773-I356-5 (pbk: alk. paper)

ISBN 978-I-4773-I357-2 (library e-book)

ISBN 978-I-4773-I358-9 (non-library e-book)

Subjects: LCSH: Street vendors-Brazil-Rio de Janeiro-History- I9th century. I Street vendors - Brazil-Rio de Janeiro-History - 2oth century. I Street vendorsBrazil-Rio de Janeiro-Social conditions. I Peddling-Brazil-Rio de JaneiroHistory - I9th century. I Peddling-Brazil-Rio de Janeiro-History-2oth century. I Slavery-Brazil-Rio de Janeiro-History. I Urban policy-Brazil-Rio de Janeiro. Classification: LCC HF5459.B6 A23 20I7 I DDC 38I/.I8098I5309034-dc23 LC record available at https://lccn.loc.gov/2016052407

doi: $10.7560 / 313558$ 
FOR ELISABETH 
THIS PAGE INTENTIONALLY LEFT BLANK 